\title{
Conservation and developmental expression of ubiquitin isopeptidases in Schistosoma mansoni
}

\author{
Roberta Verciano Pereira', Helaine Graziele Santos Vieira', \\ Victor Fernandes de Oliveira', Matheus de Souza Gomes², \\ Liana Konovaloff Jannotti Passos ${ }^{3}$, William de Castro Borges ${ }^{1}$, Renata Guerra-Sá1/+
}

\author{
${ }^{1}$ Núcleo de Pesquisas em Ciências Biológicas, Universidade Federal de Ouro Preto, Ouro Preto, MG, Brasil \\ ${ }^{2}$ Instituto de Genética e Bioquímica, Universidade Federal de Uberlândia, Campus Avançado Patos de Minas, MG, Brasil \\ ${ }^{3}$ Centro de Pesquisas René Rachou-Fiocruz, Belo Horizonte, MG, Brasil
}

Several genes related to the ubiquitin (Ub)-proteasome pathway, including those coding for proteasome subunits and conjugation enzymes, are differentially expressed during the Schistosoma mansoni life cycle. Although deubiquitinating enzymes have been reported to be negative regulators of protein ubiquitination and shown to play an important role in Ub-dependent processes, little is known about their role in $\mathrm{S}$. mansoni. In this study, we analysed the Ub carboxyl-terminal hydrolase (UCHs) proteins found in the database of the parasite's genome. An in silico analysis (GeneDB and MEROPS) identified three different UCH family members in the genome, SmUCH-L3, SmUCHL5 and SmBAP-1 and a phylogenetic analysis confirmed the evolutionary conservation of the proteins. We performed quantitative reverse transcription-polymerase chain reaction and observed a differential expression profile for all of the investigated transcripts between the cercariae and adult worm stages. These results were corroborated by low rates of Z-Arg-Leu-Arg-Gly-Gly-AMC hydrolysis in a crude extract obtained from cercariae in parallel with high Ub conjugate levels in the same extracts. We suggest that the accumulation of ubiquitinated proteins in the cercaria and early schistosomulum stages is related to a decrease in $26 S$ proteasome activity. Taken together, our data suggest that UCH family members contribute to regulating the activity of the Ub-proteasome system during the life cycle of this parasite.

Key words: deubiquitinating enzymes - ubiquitin C-terminal hydrolase - differential expression - deubiquitination activity

Ubiquitin (Ub) is a small $(\sim 8 \mathrm{kDa})$ protein that can be covalently attached to target protein substrates in a process called ubiquitination. This post-translational modification has emerged as a critical regulatory process in virtually all aspects of cell biology, including signal transduction and protein stability (Finley et al. 2004, Kerscher et al. 2006). The Ub molecule is first activated by the Ub-activating enzyme E1 and then transferred to the Ub-conjugating enzyme $\mathrm{E} 2$ and attached to a specific substrate with the assistance of Ub-ligase E3 (Hershko \& Ciechanover 1998). The covalent attachment of polyubiquitin to a protein serves as a highly specific degradation tag that mediates trafficking to the proteasome. Ub has seven internal lysine residues, any of which can by polyubiquitinated to create $\mathrm{Ub}$ chains of distinct linkages. A Ub code exists whereby proteins tagged with K6, K11, K27, K29 and K48-linked polyubiquitin chains are targeted for proteasomal degradation, whereas K63-linked chains are preferentially involved in the lysosomal pathway (Ikeda \& Dikic 2008, Dammer et al. 2011).

doi: $10.1590 / 0074-0276130107$

Financial support: FAPEMIG (CBB 0558/09), NuBio UFOP, CNPq

+ Corresponding author: rguerra@iceb.ufop.br

Received 25 February 2013

Accepted 9 September 2013
Protein ubiquitination is a reversible process involving deubiquitinating enzymes (DUBs), which are able to process linear polyubiquitin precursor proteins, such as ribosomal fusion proteins, into single $\mathrm{Ub}$ molecules (Wing 2003). Second, DUBs recycle Ub by processing polyubiquitin chains to generate free monomers that can enter the pool for subsequent conjugation events. This is a critical process because free polyubiquitin chains can inhibit the competitive binding of polyubiquitinated substrates to the $26 \mathrm{~S}$ proteasome. Finally, DUBs remove $\mathrm{Ub}$ from ubiquitinated substrates, which antagonises Ub conjugation by E3 ligases (Komander et al. 2009). DUBs have been subdivided into six families based on sequence and structural similarity: Ub-specific proteases (USPs), Ub carboxyl-terminal hydrolases (UCHs), ovarian-tumour proteases (OTUs), Machado-Joseph disease protein domain proteases, JAMM/MPN domainassociated metallopeptidases and monocyte chemotactic protein-induced protein (Fraile et al. 2012).

UCHs were the first DUB family to be structurally characterised. These enzymes only target small peptides from the $\mathrm{C}$ terminus of $\mathrm{Ub}$ because they have a confined loop that precludes the processing of polyubiquitin chains and folded target proteins (Komander et al. 2009). There are four UCHs in humans: UCH-L1, UCH-L3, UCH-L5 (UCH37) and BAP-1 (binding to the wild-type BRCA1 RING finger domain). The additional C-terminal extension present in UCH-L5 facilitates the trimming of polyubiquitin chains from conjugated proteins, whereas 
the C-terminal extension of BAP-1 interacts with the N-terminal ring finger of BRCA1 (Jensen et al. 1998, Koulich et al. 2008). Although USP-5 is a member of the USP family, this protease is an isopeptidase with four domains: a ZnF UBP domain, a USP/UBP domain and two UBA domains. USP-5 specifically disassembles unanchored polyubiquitin through its multiple Ub-binding domains (Reyes-Turcu \& Wilkinson 2009).

Schistosomes are parasitic worms that are a prime example of a complex multicellular pathogen that affects humans. The development of Schistosoma mansoni in a vertebrate host requires several coordinated alterations of its body morphology and biochemical changes that guarantee adaptation (Stirewalt 1974, Fishelson et al. 1992, McKerrow \& Salter 2002). Recently, our group showed that the Ub-proteasome proteolytic pathway in S. mansoni plays a crucial role in regulating the activity of the proteasome during parasite development (Guerra-Sá et al. 2005, Castro-Borges et al. 2007). However, despite their potential importance, little is known about DUBs in this parasite. In the present study, we identified UCH-L3, UCH-L5 (UCH37), BAP-1 and USP-5 (isopeptidase T) in S. mansoni and found that these DUBs exhibit differential gene expression profiles during development. In addition, we evaluated the activity of SmUCH-L3 and SmUSP-5 using the fluorescent substrate Z-Arg-Leu-Arg-Gly-GlyAMC and report high levels of ubiquitinated proteins in the cercaria, early schistosomulum and egg stages.

\section{MATERIALS AND METHODS}

Ethics statement - All experiments involving animals were authorised by the Ethical Committee for Animal Care of the Federal University of Ouro Preto (protocol 2011/55). These procedures were conducted in accordance with the accepted national and international regulations for laboratory animal use and care.

Parasites - The $S$. mansoni LE strain was maintained by routine passage through Biomphalaria glabrata snails and BALB/c mice. Infected snails were induced to shed cercariae under light exposure for $2 \mathrm{~h}$, followed by recovery of the larvae by sedimentation on ice. Adult worm parasites were obtained by liver perfusion of mice after infection for 50 days. The mouse livers were triturated in phosphate buffer ( $\mathrm{pH}$ 8.2); trypsin was added and the homogenate was incubated for $2.5 \mathrm{~h}$ at $37^{\circ} \mathrm{C}$ in a water bath. The eggs were recovered in saline solution after sequential sieving through 360 - and $180-\mu \mathrm{m}$ mesh. Mechanically transformed schistosomula (MTS) were prepared as described by Harrop and Wilson (1993). Briefly, cercariae were recovered and washed in RPMI1640 medium (Invitrogen, São Paulo, Brazil) and then vortexing at maximum speed for $90 \mathrm{~s}$; the cercariae were immediately cultured for $3.5 \mathrm{~h}$ at $37^{\circ} \mathrm{C}$ and $5 \% \mathrm{CO}_{2}$. The recovered schistosomula were washed with RPMI-1640 until no tails were detected. For the subsequent incubations, the parasites were maintained in M169 medium supplemented with $10 \%$ foetal bovine serum, penicillin and streptomycin $(100 \mu \mathrm{g} / \mathrm{mL})$ and $5 \%$ Schneider's medium (Basch \& DiConza 1977 ) at $37^{\circ} \mathrm{C}$ with $5 \% \mathrm{CO}_{2}$ for $3.5 \mathrm{~h}, 24 \mathrm{~h}, 48 \mathrm{~h}$ and $72 \mathrm{~h}$ and 5, 8 and 10 days.
Analysis of UCH genes - UCH genes were identified by mining $S$. mansoni sequences in the GeneDB (genedb.org/genedb/smansoni/) and MEROPS (merops. sanger.ac.uk/) databases (Rawlings et al. 2008, 2010) using BLASTp and queries of known Homo sapiens proteins (BAP1, GeneDB ID: NP 004647.1 and MEROPS ID: MER003989; UCH-L3, GeneDB ID: NP_005993.1 and MEROPS ID: MER000836; UCH-L5, GeneDB ID: NP_057068.1 and MEROPS ID: MER005539). Reference proteins from other species were searched in the National Center for Biotechnology Information database to obtain a full set of putative homologue proteins to compare with the S. mansoni proteins. The BLASTp algorithm, underpinned by Pfam (v26.0), allowed for the detection of conserved protein domains or motifs from S. mansoni sequences. The entire protein sequences were used to perform multiple sequence alignments using the programme CLUSTALX 2.0 with the default settings (Larkin et al. 2007). Phylogenetic trees were inferred using the neighbour-joining method and the Jones-TaylorThornton model (Saitou \& Nei 1987). A bootstrap consensus tree inferred from 1,000 replicates was used to represent the evolutionary history of the taxa analysed. The molecular phylogenetic analyses were conducted using MEGA 5 software (Tamura et al. 2011). The sequences used as queries obtained from the $H$. sapiens database, i.e., UCH-L5 (Li et al. 2001, Nishio et al. 2009, Maiti et al. 2011, Burgie et al. 2012), UCH-L3 (Johnston et al. 1997, Larsen et al. 1998) and BAP-1 (Sanchez-Pulido et al. 2012), were experimentally supported.

Expression analysis of DUB enzymes - Total RNA from cercariae, schistosomula, adult worms and eggs was obtained using a combination of the Trizol reagent (Sigma, Belo Horizonte, MG, Brazil) and chloroform for extraction and the RNA was column-purified using the SV total RNA Isolation system (Promega, Belo Horizonte, MG, Brazil). The preparation was treated with RNase-free DNase I in three different rounds with decreasing enzyme concentrations (RQ1 DNase; Promega). The RNA was quantified using a spectrophotometer and an aliquot containing $1 \mu \mathrm{g}$ of total RNA was reverse transcribed using an oligo dT primer from the Thermoscript reverse transcription-polymerase chain reaction (RT-PCR) System (Invitrogen), as described by the manufacturer. The efficiency of DNAse I treatment was evaluated by PCR amplification of the cDNA reaction mix without the addition of the ThermoScript enzyme. S. mansoni-specific primers were designed using the programme GeneRunner ${ }^{\circledR}$. The sequence accessions and primer pairs are shown in Supplementary data. RT-cDNA samples were used as the templates for PCR amplification with the SYBR Green Master Mix UDG-ROX $^{\circledR}$ (Invitrogen) and 7300 Real-time PCR System (Applied Biosystems, Rio de Janeiro, Brazil). Specific primers for $S$. mansoni EIF4E were used as an endogenous control (GeneDB ID: Smp_001500) (forward, 5'TGTTCCAACCACGGTCTCG3'; reverse, 5'TCGCCTTCCAATGCTTAGG3') (Liu et al. 2012). The efficiency of each pair of primers was evaluated according to the protocol developed by Applied Biosystems 
(cDNA dilutions 1:10, 1:100, 1:1000). For all investigated transcripts, three biological replicates were performed and gene expression was normalised against the EIF4E transcript according to the $2^{-\Delta \mathrm{Ct}}$ method (Livak \& Schmittgen 2001) using Applied Biosystems 7300 software.

In vitro activity assay - To determine the enzymatic activity of the proteases present in the crude extracts from adult worms, cercariae, eggs and schistosomula, we used the fluorogenic peptide substrate Z-Arg-LeuArg-Gly-Gly-AMC (Sigma), which is specific for assaying UCH-L3 and USP-5 activities. In these assays, 30 $\mu \mathrm{g}$ of total protein was used with $13 \mu \mathrm{M}$ of the peptide substrate in $50 \mathrm{mM}$ Tris- $\mathrm{HCl}(\mathrm{pH} 7.5), 5 \mathrm{mM} \mathrm{MgCl}, 1$ $\mathrm{mM}$ dithiothreitol (DTT) and $\pm 50 \mu \mathrm{M}$ n-ethylmaleimide (NEM) (Sigma) to control for specific enzyme inhibition. Each enzymatic assay was performed in a final volume of $100 \mu \mathrm{L}$ and the reaction proceeded for $30 \mathrm{~min}$ at $37^{\circ} \mathrm{C}$. Peptide hydrolysis was stopped by the addition of $2 \mathrm{~mL}$ of $99.5 \%$ ethanol. The fluorescence was measured at $380 \mathrm{~nm}$ (excitation) and $440 \mathrm{~nm}$ (emission) using a spectrofluorimeter (Turner QuantechTM Fluorimeter) and the results are expressed in fluorescence units per $\mu \mathrm{g}$ of total protein.

Detection of ubiquitinated proteins by western blotting $(W B)$ - Ubiquitinated proteins were identified by WB using a polyclonal anti-Ub (Sigma) antibody. Brief- ly, total protein extracts from cercariae, schistosomula at $3.5 \mathrm{~h}, 5 \mathrm{~d}, 8 \mathrm{~d}$ and $10 \mathrm{~d}$, adult worms and eggs were prepared by sonication in $25 \mathrm{mM}$ Tris- $\mathrm{HCl}(\mathrm{pH} \mathrm{7.5)}$, $1 \mathrm{mM}$ DTT, $1 \mathrm{mM}$ ethylenediamine tetraacetic acid and $10 \mu \mathrm{M}$ of the following protease inhibitors: TLCK, TPCK, NEM and PMSF. After centrifugation at 10,000 $g$ for $30 \mathrm{~min}$, the soluble protein content was determined using the QuantiPro ${ }^{\mathrm{TM}}$ BCA Assay Kit (Sigma). A $20-\mu \mathrm{g}$ sample of total soluble protein was separated by $12 \%$ sodium dodecyl sulfate polyacrylamide gel electrophoresis and the proteins were transferred to a PVDF membrane at $25 \mathrm{~V}$ for $2 \mathrm{~h}$ at $4^{\circ} \mathrm{C}$. After $16 \mathrm{~h} \mathrm{incu-}$ bation in blocking solution, the membrane was washed and then incubated with a primary anti-Ub antibody at a 1:1,000 dilution. Peroxidase-conjugated anti-mouse IgG (Sigma) was used, as the secondary antibody at 1:2,000, and the reactivity was visualised using ECL Western blotting reagents (GE Healthcare, UK) according to the manufacturer's instructions.

Statistical analysis - A statistical analysis was performed using GraphPad Prism version 5.0 (Irvine, CA, USA). The normality of the data was established using an ANOVA and Tukey post-tests were used to investigate the significance of the differential expression of transcripts during the investigated stages. In all cases, the differences were considered significant when $\mathrm{p}<0.05$.

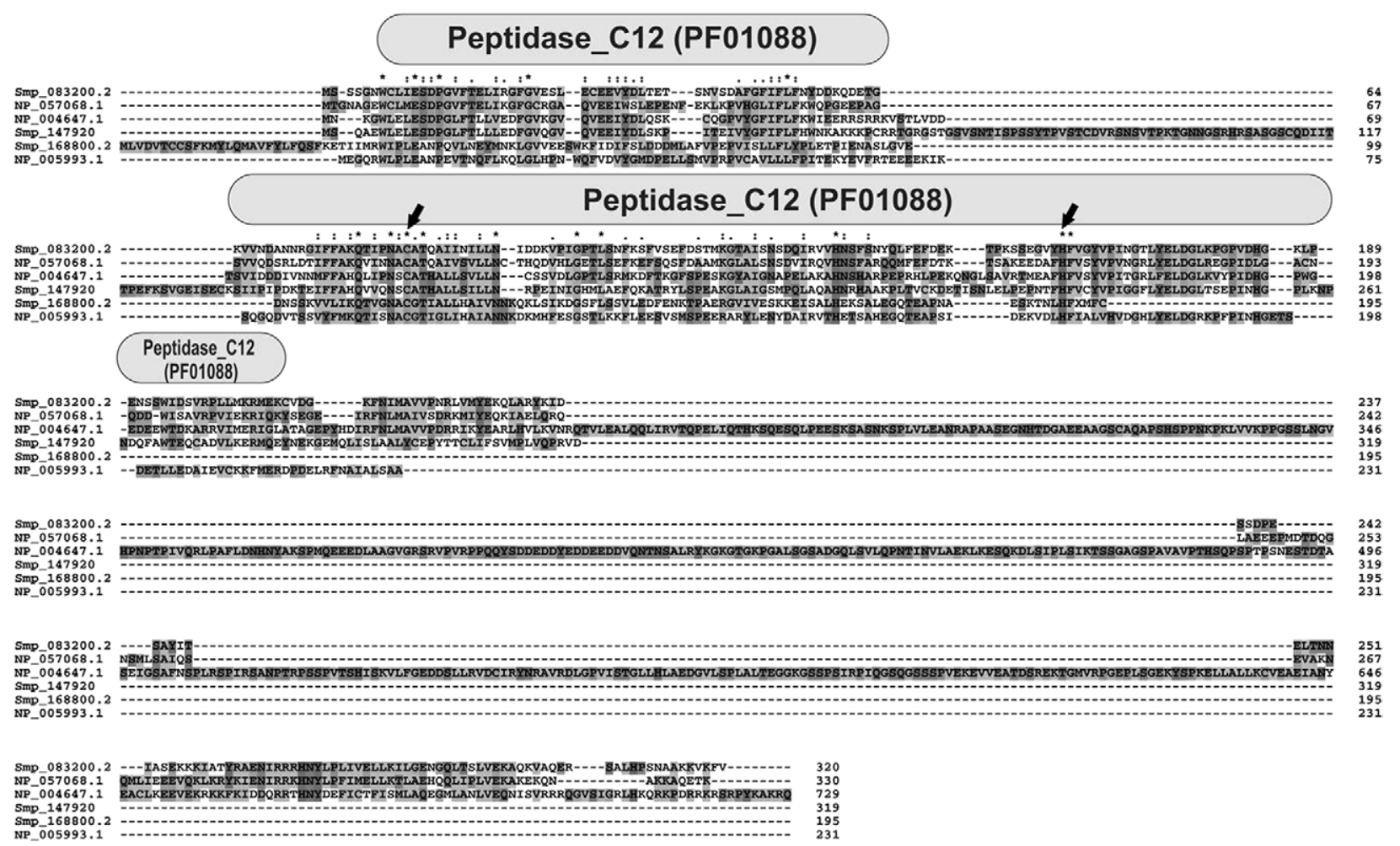

Fig. 1: Schistosoma mansoni and Homo sapiens ubiquitin carboxy-terminal hydrolases (UCHs) alignment. UCH-L3, UCH-L5 and BAP-1 alignment using CLUSTALX 2.0. The domain position of the UCH (Pfam: PF01088, Peptidase_C12) in S. mansoni and H. sapiens were based on the Pfam database. The grey boxes represent the conserved domain. Aligned catalytic residues are denoted by arrows. Asterisk indicates identical residues, highly conserved amino acid substitution and conserved amino acid substitutions, respectively. 


\section{RESULTS}

Conservation of UCH in S. mansoni - An in silico analysis revealed six sequences representing three putative $\mathrm{UCH}$ sequences in the $S$. mansoni genome: Smp_168800.1/2/4 (UCH-L3), Smp_083200.1/2 (UCHL5) and (BAP-1). Three similar sequences for UCHL3 were found, which are most likely the result of alternative splicing and are annotated as Smp_168800.1, Smp_168800.2 and Smp_168800.4. Among them, the Smp_168800.2 sequence showed an insertion of 43 amino acids in the N-terminal region of the protein sequence. We also observed a putative alternative splicing event for UCH-L5, with two similar sequences that were annotated as Smp 083200.1 and Smp 083200.2.

Analyses using the Pfam protein domain database (v26.0) were performed to confirm whether these predicted proteases can be considered to be cysteine-protease family members. For all UCH entries, a conserved domain (Pfam: PF01088, Peptidase_C12) was identified in the three $S$. mansoni UCH homologues (Fig. 1). The SmUCH-L3, SmUCH-L5 and SmBAP-1 sequences contain two catalytic residues represented by a cysteine at

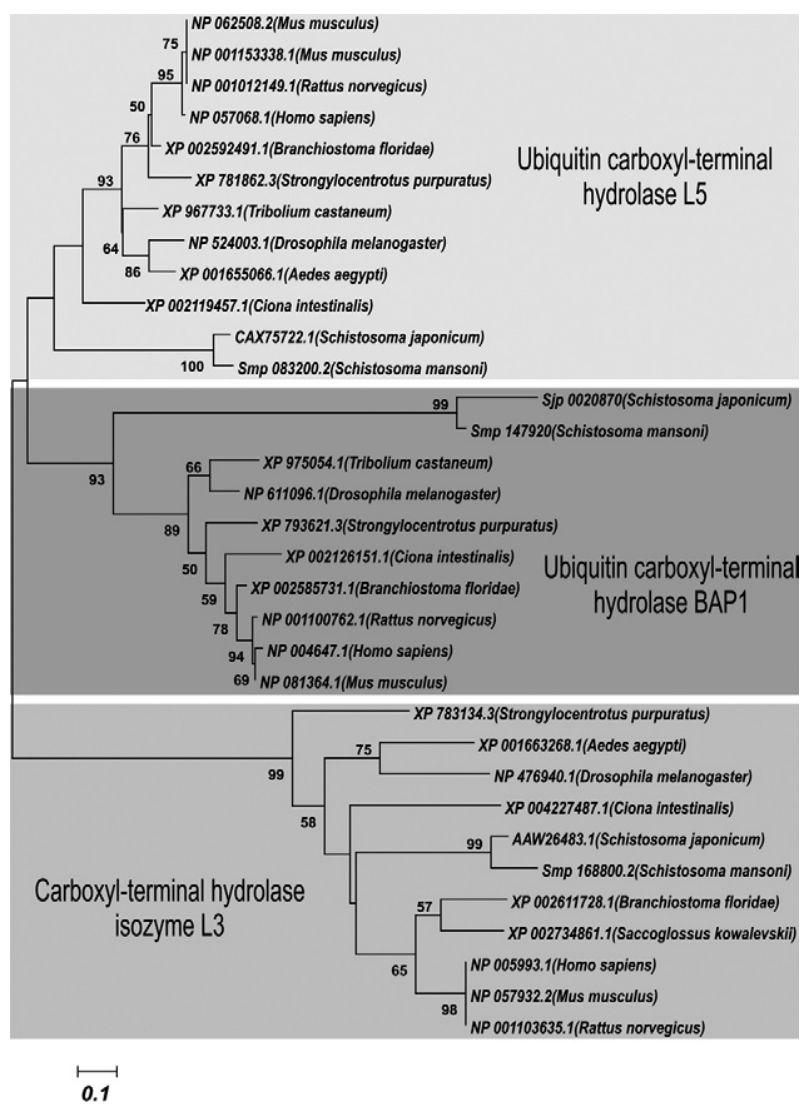

Fig. 2: consensus phylogenetic tree based on amino acid sequences of SmUCH-L3, SmUCH-L5 and SmBAP-1. Tree construction and bootstrap analysis were performed using CLUSTALX 2.0 and MEGA 5.0. For the consensus tree and reliability of the branches formed, a phylogenetic bootstrap analysis using 1,000 replicates for each sequence was used with a $50 \%$ the minimum for considering branch reliably. positions 115,85 and 153 , respectively, and a histidine at positions 189,160 and 231, respectively. We observed that UCH-L5 showed high similarity $(78 \%)$ and UCHL3 and BAP-1 showed low similarities $(55 \%$ and $26 \%$, respectively) to their human orthologues.

We also evaluated the conservation of these proteins during evolution using a phylogenetic analysis. Our results showed that each UCH was grouped into a distinct clade close to Schistosoma japonicum, which reinforces the structural conservation among the orthologues (Fig. 2). Given the high sequence similarity, the three $S$. mansoni proteases were grouped in the same branch of putative UCH proteins from $S$. japonicum. Furthermore, it was noted that UCH-L3 occupies a distinct clade relative to the other two UCHs, BAP-1 and UCH-L5. Indeed, the UCH-L3 sequences are more closely related, whereas SmUCH-L5 and SmBAP-1 show divergence from their respective orthologues.

DUB enzymes are differentially expressed in $S$. mansoni - The gene expression profiles of Smuch-l3, Smuch-l5, Smbap-1 and Smusp 5 were determined using quantitative RT-PCR during the cercaria-schistosomulum transition and also in adult worms and eggs (Fig. 3). We observed that Smuch-l3, Smuch-15, Smbap-1 and Smusp5 transcripts were expressed at basal levels in cercariae. Conversely, the level of Smuch-l3 transcript was significantly higher in adult worms and was approximately five and three-fold higher in MTS-3.5 h compared to the levels in cercariae and during schistosomulum development (MTS-24 h, MTS-48 h and MTS-72 h), respectively. With regard to Smuch-15, we observed lower expression in cercariae (2-fold) compared to schistosomula, adult worms and eggs. Furthermore, a two-fold difference was observed in adult worms when compared to the other stages and Smbap-1 transcripts were also less abundant during this stage of the parasite's life cycle. The levels of Smusp-5 transcripts were significantly higher (3-fold) in the early schistosomulum compared to the other stages.

SmUCH-L3 and SmUSP-5 enzyme activities - In vitro endopeptidase assays were performed using crude extracts from cercariae, schistosomula, adult worms and eggs (Table). The UCH-L3 and USP-5 enzyme activities were slightly higher in the MTS-72 $\mathrm{h}$ and adult worms compared to cercariae and eggs. The enzyme activities were also measured in the presence of a commercially available UCH inhibitor, NEM and significant differences were observed in all the analysed stages, which were at least two-fold lower relative to the extracts without the inhibitor.

Ub conjugates in $S$. mansoni - To confirm the presence of Ub conjugates in the parasite, WB was used to examine crude extracts from cercariae, MTS-3.5 h, MTS- $5 \mathrm{~d}$, MTS- $8 \mathrm{~d}$, MTS-10 d, adult worms and eggs. The molecular mass distribution of Ub conjugates is presented in Fig. 4 , with the majority of the conjugates appearing within the mass range of $20-100 \mathrm{kDa}$. It was observed that $\mathrm{Ub}$ conjugates accumulate in the cercariae and early schistosomula though appear at low levels in late schistosomula 
and adult worms. In contrast, the egg stage exhibited the most pronounced increase in the levels of $\mathrm{Ub}$ conjugates relative to all the other stages examined.

\section{DISCUSSION}

DUBs directly regulate the protein ubiquitination process. Most commonly, ubiquitination leads to protein degradation, whereas deubiquitination has a stabilising effect and actively increases protein levels in cells. In addition, these enzymes are involved in numerous biological processes, such as growth, differentiation and transcriptional regulation (Sridhar et al. 2007, Singhal et al. 2008, Fraile et al. 2012). Given that protein homeostasis and cell signalling often require tight temporal and spatial regulation, the DUBs affecting these pathways are also regulated in many different ways. Previous results from our group have demonstrated that the accumulation of ubiquitinated conjugates in cercariae correlated with decreased $26 \mathrm{~S}$ proteasome activity at this stage (GuerraSá et al. 2005, Pereira-Júnior et al. 2012).

UCHs catalyse the hydrolysis of $\mathrm{Ub}$ in the C-terminal region and these enzymes play a key role in the maturation of $\mathrm{Ub}$ by processing its precursor in addition to recycling the free monomer by disassembling polyubiquitin chains (Fang et al. 2010). In the present study, the genes coding for at least three UCHs (UCH-L3, UCH-L5 and BAP-1) were identified in the S. mansoni genome. In contrast, our in silico analyses did not reveal the presence of UCH-L1, a hydrolase that has been implicated in Parkinson's disease and neuronal function in humans (Reyes-Turcu \& Wilkinson 2009). Although the specificity and function of UCHs in parasite biology remain elusive, each of these enzymes contains conserved catalytic domains (UCH-domains) that are critical for enzymatic activity. In addition, the SmUCHs domain contains two conserved cysteine and histidine residues, which constitute a signature for all UCH family members. Subsequent reports concerning the crystal structures of UCH-L3 and UCH-L5 have demonstrated three conserved residues (cysteine, histidine and aspartate) in UCH-domains (Johnston et al. 1997, Nishio et al. 2009). UCH-L5 and BAP-1, which are more similar to each other and have diverged from UCH-L3, occupy a distinct subclade and share a common ancestor (Sanchez-Pulido et al. 2012). This observation is corroborated by the fact that UCH-L5 and BAP-1 share 60 amino acids at their Cterminus (approximately $38 \%$ ), which is designated the UCH37 like-domain (Eletr \& Wilkinson 2011).

UCH-L5 can suppress proteasome-mediated degradation via the disassembly of distal polyubiquitin moieties (Lam et al. 1997, Koulich et al. 2008, Schreiner et al. 2008). However, a recent study supports the role of UCH-L5 in catalysing the selected degradation of specific proteasome substrates, such as nitric oxide synthase and IкB (Mazumdar et al. 2010). These findings raise the possibility that $\mathrm{UCH}-\mathrm{L} 5$ has a double regulatory role

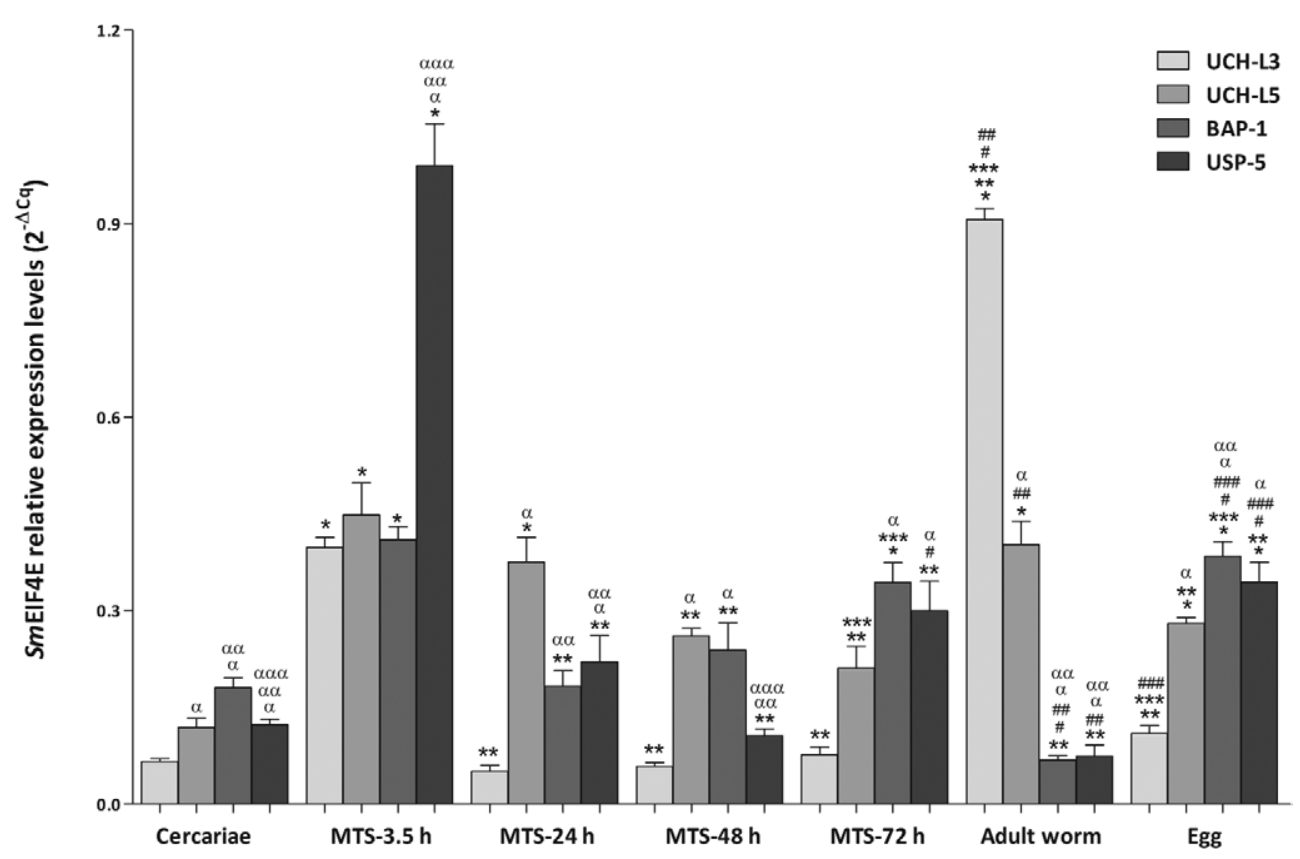

Fig. 3: SmUCH-L3, SmUCH-L5, SmBAP-1 and SmUSP-5 are differentially expressed throughout the Schistosoma mansoni life cycle. SmUCHL3, SmUCH-L, SmBAP-1 and SmUSP-5 mRNA expression levels were measured based on three replicates for each of the following stages: adult worms, cercariae and eggs as well as mechanically transformed schistosomula (MTS)-3.5 h, $24 \mathrm{~h}, 48 \mathrm{~h}$ and $72 \mathrm{~h}$ using quantitative reverse transcription-polymerase chain reaction. Expression levels were calibrated according to the comparative $2^{-\Delta \mathrm{Ct}}$ method using the constitutively expressed SmEIF4E as an endogenous control (ANOVA followed by Tukey's pairwise comparison p < 0.05). *: different from cercariae; **: different from MTS-3.5 h; ***: different from MTS-24 h; \#: different from MTS-48 h; \#\#: different from MTS-72 h; \#\#\#: different from adult worm; ${ }^{\alpha}$ : different from ubiquitin (Ub) carboxy-terminal hydrolases (UCH)-L3, ${ }^{\alpha \alpha}$ : different from UCH-L5; ${ }^{\alpha \alpha}$ : different from BAP-1; USP: Ub-specific proteases. 
TABLE

SmUCH-L3 and SmUSP-5 enzymatic activities in Schistosoma mansoni

\begin{tabular}{lccccccc}
\hline $\begin{array}{l}\text { Deubiquitinating } \\
\text { activity }\end{array}$ & Cercariae & MTS-3.5 h & MTS-24 h & MTS-48 h & MTS-72 h & Adult worms & Eggs \\
\hline NEM (-) & $110 \pm 0.46$ & $300 \pm 0.87$ & $315 \pm 0.87$ & $150 \pm 0.58$ & $835 \pm 1.04$ & $833 \pm 32.59$ & $270 \pm 0.58$ \\
NEM (+) & $8 \pm 0.01$ & $90 \pm 0.29$ & $70 \pm 0.29$ & $45 \pm 0.17$ & $169 \pm 0.87$ & $150 \pm 0.46$ & $85 \pm 0.52$
\end{tabular}

the fluorescence levels were measured based on three replicates. Statistical analysis was performed using t tests followed by the unpaired test $\mathrm{p}<0.001$. The fluorescence was expressed as indicated (see Materials and Methods). The results represent the average of three independent experiments \pm standard error of the means. MTS: mechanically transformed schistosomula; UCH: ubiquitin (Ub) carboxy-terminal hydrolases; USP: Ub-specific proteases.

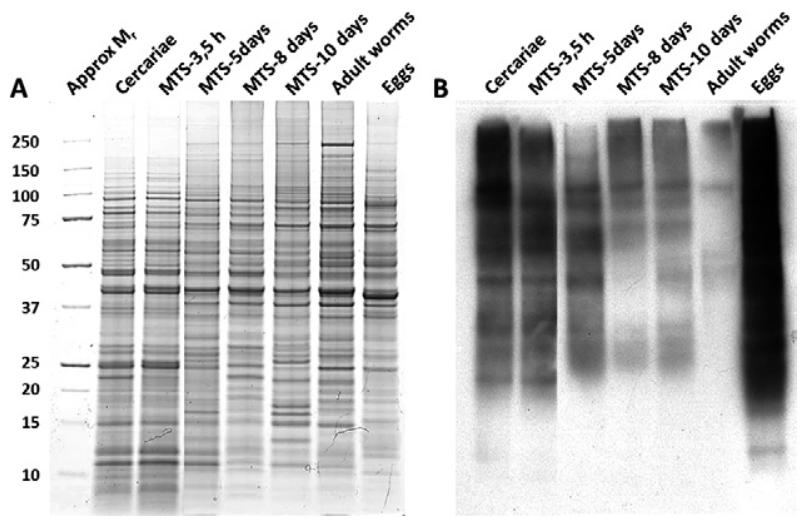

Fig. 4: the ubiquitination profile throughout the Schistosoma mansoni life cycle. A: gel stained in Sypro Ruby ${ }^{\mathbb{}}$; B: $10 \mu \mathrm{g}$ of crude extracts were analysed by $12 \%$ sodium dodecyl sulfate polyacrylamide gel electrophoresis followed by immunoblotting using anti-ubiquitin antibody produced in rabbit.

in suppressing or promoting the degradation of specific proteasome substrates. The DUB activity of UCH-L5 is tightly regulated and is dependent on its association with the Ub receptor Rpn13/ADRM1, which is located at the base of 19S RP (Hamazaki et al. 2006, Qiu et al. 2006, Yao et al. 2006, Lander et al. 2012). Although UCH-L5 can cleave polyubiquitin when bound to the proteasome, this reaction may require the participation of additional proteasome components to partially unfold the polyubiquitin chain (Eletr \& Wilkinson 2011). We detected low levels of UCH-L5 expression in the cercaria stage, in agreement with the previous results from our group that demonstrated decreased $26 \mathrm{~S}$ proteasome activity in extracts from cercariae relative to adult worms (PereiraJúnior et al. 2012).

BAP-1, a tumour suppressor, is a nuclear DUB with an N-terminal UCH domain and two predicted nuclear localisation signals (NLSs), though only one is a classic NLS (Ventii et al. 2008). BAP-1 has been reported to influence cell proliferation at G1/S (Eletr \& Wilkinson 2011). Smbap-1 transcripts were up-regulated in MTS$3.5 \mathrm{~h}, \mathrm{MTS}-72 \mathrm{~h}$ and eggs and are down-regulated in cercariae and adult worms, results that are consistent with a possible participation of SmBAP-1 in parasite stages during which cell proliferation is particularly required, as observed for the larval stage (Stirewalt 1974, Fishelson et al. 1992, McKerrow \& Salter 2002).

We found that Smuch-l3 transcripts were up-regulated in MTS-72 h, adult worms and eggs and down-regulated in cercariae. UCH-L3 is a Ub-protein hydrolase that is involved in the processing of $\mathrm{Ub}$ precursors and ubiquitinated substrates to generate free Ub. The enzyme recognises and hydrolyses isopeptide bonds at the C-terminal glycine of either Ub or Nedd8, a Ub-like protein (Hemelaar et al. 2004, Frickel et al. 2007). Although neither dimerisation nor ligase activity is observed for UCH-L3, this hydrolase can interact with Lys48-linked Ub dimers to protect it from degradation and inhibit its hydrolase activity (Fang et al. 2010). SmUCH-L3 was highly expressed in adult worms, suggesting an important role at this stage.

A unique USP enzyme analysed in this study was USP-5, which is also an isopeptidase. The transcript levels of this protease showed higher expression in MTS-3.5 $\mathrm{h}$ and similar levels in cercariae and adult worms. USP-5 is responsible for the majority of unanchored polyubiquitin disassembly (Eletr \& Wilkinson 2011) and another significant finding of this study was the correlation between the expression and activity of SmUCH-L3 and SmUSP-5. Although the fluorogenic substrate utilised does not allow the discrimination between UCH-L3 and USP-5 activities, it is likely that the peptide hydrolysis observed in the cercariae was strongly related to UCHL3, given its higher expression at this stage.

The WB analysis for the detection of ubiquitinated proteins in $S$. mansoni revealed the characteristic band smearing at all investigated stages, possibly reflecting proteins tagged with a varying number of Ub molecules (Carlson et al. 1987, Pickart et al. 1991). As each Ub moiety adds approximately $8 \mathrm{kDa}$ to the target protein conjugate, the length of the Ub chain influences the apparent molecular mass of Ub conjugates. The accumulation of ubiquitinated conjugates in cercariae compared to adult worms is consistent with the results obtained by GuerraSá et al. (2005), showing the lowest activity of the 26S proteasome. Moreover, the most intense accumulation of ubiquitinated conjugates was observed in the egg stage. In this regard, Mathieson et al. (2011) showed development and morphology-specific characteristics of the proteasome-Ub pathway in S. mansoni eggs. 
In summary, our study addresses the biological role played by this particular UCH sub-family during the $S$. mansoni life cycle and our results raise a number of questions concerning the regulation of proteasome activity and its role in schistosome biology. The recombinant versions of these proteins might allow for the assessment their substrate specificity and specific anti-SmUCHs antibodies could provide a deeper understanding of the role of these enzymes in this parasite.

\section{ACKNOWLEDGEMENTS}

To transcriptome initiatives: São Paulo Transcriptome Consortium, Minas Gerais Genome Network and Welcome Trust Genome Initiative (UK).

\section{REFERENCES}

Basch P, DiConza J 1977. In vitro development of Schistosoma mansoni cercariae. J Parasitol 63: 245-249.

Burgie SE, Bingman CA, Soni AB, Phillips Jr GN 2012. Structural characterization of human Uch37. Proteins 80: 649-654.

Carlson N, Rogers S, Rechsteiner M 1987. Microinjection of ubiquitin: changes in protein degradation in HeLa cells subjected to heat-shock. J Cell Biol 104: 547-555.

Castro-Borges W, Cartwright J, Ashton PD, Braschi S, Guerra-Sá R, Rodrigues V, Wilson RA, Curwen RS 2007. The 20S proteasome of Schistosoma mansoni: a proteomic analysis. Proteomics 7: 1065-1075.

Dammer CH, Na P, Xu NT, Seyfried DM, Duong D, Cheng D, Gearing M, Rees H, Lah JJ, Levey AI, Rush J, Peng J 2011. Polyubiquitin linkage profiles in three models of proteolytic stress suggest the etiology of Alzheimer disease. J Biol Chem 286: 10457-10465.

Eletr ZM, Wilkinson KD 2011. An emerging model for BAP1's role in regulating cell cycle progression. Cell Biochem Biophys 60: 3-11.

Fang Y, Fu D, Shen XZ 2010. The potential role of ubiquitin c-terminal hydrolases in oncogenesis. Biochim Biophys Acta 1806: 1-6.

Finley D, Ciechanover A, Varshavsky A 2004. Ubiquitin as a central cellular regulator. Cell 116 (Suppl. 2) S29-S32.

Fishelson Z, Amiri P, Friend DS, Marikovsky M, Petitt M, Newport G, Mckerow JH 1992. Schistosoma mansoni: cell-specific expression and secretion of a serine protease during development of cercariae. Exp Parasitol 75: 87-98.

Fraile JM, Quesada V, Rodríguez D, Freije JM, López-Otín C 2012. Deubiquitinases in cancer: new functions and therapeutic options. Oncogene 31: 2373-2388.

Frickel EM, Quesada V, Muething L, Gubbels MJ, Spooner E, Ploegh H, Artavanis-Tsakonas K 2007. Apicomplexan UCHL3 retains dual specificity for ubiquitin and Nedd8 throughout evolution. Cell Microbiol 9: 1601-1610.

Guerra-Sá R, Castro-Borges W, Evangelista EAB, Kettelhut IC, Rodrigues V 2005. Schistosoma mansoni: functional proteasomes are required for development in the vertebrate host. Exp Parasitol 109: 228-236.

Hamazaki J, Iemura S, Natsume T, Yashiroda H, Tanaka K, Murata $\mathrm{S} 2006$. A novel proteasome interacting protein recruits the deubiquitinating enzyme $\mathrm{UCH} 37$ to $26 \mathrm{~S}$ proteasomes. EMBO J 25: 4524-4536.

Harrop R, Wilson RA 1993. Protein synthesis and release by cultured schistosomula of Schistosoma mansoni. Parasitology 107: 265-274.

Hemelaar J, Borodovsky A, Kessler BM, Reverter D, Cook J, Kolli N, Gan-Erdene T, Wilkinson KD, Gill G, Lima CD, Ploegh HL,
Ovaa H 2004. Specific and covalent targeting of conjugating and deconjugating enzymes of ubiquitin-like proteins. Mol Cell Biol 24: 84-95.

Hershko A, Ciechanover A 1998. The ubiquitin system. Annu Rev Biochem 67: 425-479.

Ikeda F, Dikic I 2008. Atypical ubiquitin chains: new molecular signals, protein modifications: beyond the usual suspects' review series. EMBO Rep 9: 536-542.

Jensen DE, Proctor M, Marquis ST, Gardner HP, Ha SI, Chodosh LA, Ishov AM, Tommerup N, Vissing H, Sekido Y, Minna J, Borodovsky A, Schultz DC, Wilkinson KD, Maul GG, Barlev N, Berger SL, Prendergast GC, Rauscher III FJ 1998. BAP1: a novel ubiquitin hydrolase which binds to the BRCA1 RING finger and enhances BRCA1-mediated cell growth suppression. Oncogene 16: 1097-1112.

Johnston SC, Larsen CN, Cook WJ, Wilkinson KD, Hill CP 1997. Crystal structure of a deubiquitinating enzyme (human UCH-L3) at 1.8 A resolution. EMBO J 16: 3787-3796.

Kerscher O, Felberbaum R, Hochstrasser M 2006. Modification of proteins by ubiquitin and ubiquitin-like proteins. Annu Rev Cell Dev Biol 22: 159-180.

Komander D, Clague MJ, Urbe S 2009. Breaking the chains: structure and function of the deubiquitinases. Nat Rev Mol Cell Biol 10: $550-563$.

Koulich E, Li X, Demartino GN 2008. Relative structural and functional roles of multiple deubiquitylating proteins associated with mammalian 26S proteasome. Mol Biol Cell 19: 1072-1082.

Lam YA, DeMartino GN, Pickart CM, Cohen RE 1997. Specificity of the ubiquitin isopeptidase in the PA700 regulatory complex of 26S proteasomes. J Biol Chem 272: 28438-28446.

Lander GC, Estrin E, Matyskiela ME, Bashore C, Nogales E, Martin A 2012. Complete subunit architecture of the proteasome regulatory particle. Nature 482: 186-191.

Larkin MA, Blackshields G, Brown NP, Chenna R, McGettigan PA, McWilliam H, Valentin F, Wallace IM, Wilm A, Lopez R, Thompson JD, Gibson TJ, Higgins DG 2007. CLUSTALW and CLUSTALX version 2.0. Bioinformatics 23: 2947-2948.

Larsen CN, Krantz BA, Wilkinson KD 1998. Substrate specificity of deubiquitinating enzymes: ubiquitin C-terminal hydrolases. Biochemistry 37: 3358-3368.

Li T, Duan W, Yang H, Lee MK, Mustafa FB, Lee BH, Teo TS 2001. Identification of two proteins, S14 and UIP1, that interact with UCH37. FEBS Lett 488: 201-205.

Liu S, Cai P, Hou N, Piao X, Wang H, Hung T, Chen Q 2012. Genome-wide identification and characterization of a panel of house-keeping genes in Schistosoma japonicum. Mol Biochem Parasitol 182: 75-82.

Livak KJ, Schmittgen TD 2001. Analysis of relative gene expression data using real-time quantitative PCR and the 2(-Delta DeltaC(T)) method. Methods 25: 402-408.

Maiti TK, Permaul M, Boudreaux DA, Mahanic C, Mauney S, Das C 2011. Crystal structure of the catalytic domain of UCHL5, a proteasome-associated human deubiquitinating enzyme, reveals an unproductive form of the enzyme. FEBS J 278: 4917-4926.

Mathieson W, Castro-Borges W, Wilson RA 2011. The proteasomeubiquitin pathway in the Schistosoma mansoni egg has development- and morphology-specific characteristics. Mol Biochem Parasitol 175: 118-125.

Mazumdar T, Gorgun FM, Sha Y, Tyryshkin A, Zeng S, HartmannPetersen R, Jørgensen JP, Hendil KB, Eissa NT 2010. Regula- 
tion of NF-kappaB activity and inducible nitric oxide synthase by regulatory particle non-ATPase subunit 13 (Rpn13). Proc Natl Acad Sci USA 107: 13854-13859.

McKerrow JH, Salter J 2002. Invasion of skin by Schistosoma cercariae. Trends Parasitol 18: 193-195.

Nishio K, Kim SW, Kawai K, Mizushima T, Yamane T, Hamazaki J, Murata S, Tanaka K, Morimoto Y 2009. Crystal structure of the de-ubiquitinating enzyme UCH37 (human UCH-L5) catalytic domain. Biochem Biophys Res Commun 390: 855-860.

Pereira-Júnior OS, Pereira RV, Silva CS, Castro-Borges W, Sá RG, Cabral FJ, Silva SH, Soares CS, Morais ER, Moreira EB, Magalhães LG, de Paula FM, Rodrigues V 2012. Investigation on the 19S ATPase proteasome subunits (Rpt1-6) conservation and their differential gene expression in Schistosoma mansoni. Parasitol Res 112: 235-242.

Pickart CM, Summers RG, Shim H, Kasperek EM 1991. Dynamics of ubiquitin pools in developing sea urchin embryos. Dev Growth Differ 33: 587-598.

Qiu XB, Ouyang SY, Li CJ, Miao S, Wang L, Goldberg AL 2006. hRpn13/ADRM1/GP110 is a novel proteasome subunit that binds the deubiquitinating enzyme, UCH37. EMBO J 25: 5742-5753.

Rawlings ND, Barrett AJ, Bateman A 2010. MEROPS: the peptidase database. Nucleic Acids Res 38: 227-233.

Rawlings ND, Morton FR, Kok CY, Kong J, Barrett AJ 2008. MEROPS: the peptidase database. Nucleic Acids Res 36: 320-325.

Reyes-Turcu FE, Wilkinson KD 2009. Polyubiquitin binding and disassembly by deubiquitinating enzymes. Chem Rev 109: 1495-1508.

Saitou N, Nei M 1987. The neighbour-joining method: a new method for reconstructing phylogenetic trees. Mol Biol Evol 4: 406-425.
Sanchez-Pulido L, Kong L, Ponting CP 2012. A common ancestry for BAP1 and Uch37 regulators. Bioinformatics 28: 1953-1956.

Schreiner P, Chen X, Husnjak K, Randles L, Zhang N, Elsasser S, Finley D, Dikic I, Walters KJ, Groll M 2008. Ubiquitin docking at the proteasome through a novel pleckstrin-homology domain interaction. Nature 453: 548-552.

Singhal S, Taylor MC, Baker RT 2008. Deubiquitylating enzymes and disease. BMC Biochem 9: S3.

Sridhar VV, Kapoor A, Zhang K, Zhu J, Zhou T, Hasegawa PM, Bressan RA, Zhu JK 2007. Control of DNA methylation and heterochromatic silencing by histone H2B deubiquitylation. Nature 477: 735-738.

Stirewalt MA 1974. Schistosoma mansoni: cercaria to schistosomule. Adv Parasitol 12: 115-182.

Tamura K, Peterson D, Peterson N, Stecher G, Nei M, Kumar S 2011. MEGA5: Molecular Evolutionary Genetics Analysis using maximum likelihood, evolutionary distance and maximum parsimony methods. Mol Biol Evol 28: 2731-2739.

Ventii KH, Devi NS, Friedrich KL, Chernova TA, Tighiouart M, van Meir EG, Wilkinson KD 2008. BRCA1-associated protein 1 is a tumor suppressor that requires deubiquitinating activity and nuclear localization. Cancer Res 68: 6953-6962.

Wing SS 2003. Deubiquitinating enzymes-the importance of driving in reverse along the ubiquitin-proteasome pathway. Int $J$ Biochem Cell Biol 35: 590-605.

Yao T, Song L, Xu W, DeMartino GN, Florens L, Swanson SK, Washburn MP, Conaway RC, Conaway JW, Cohen RE 2006. Proteasome recruitment and activation of the Uch37 deubiquitinating enzyme by Adrm1. Nat Cell Biol 8: 994-1002. 
Sequence accessions and the primer pairs

\begin{tabular}{lccc}
\hline Genes & GeneDB ID & MEROPS ID & Primers \\
\hline Smuch-13 & Smp_168800.2 & MER237456 & F: 5'CGCTGCTCAAGAAAGAAC3' \\
& & R: 5'TTGGGTGTCGAGGATAAC3' \\
Smuch-15 & Smp_083200.2 & MER185278 & F: 5'CCGAAACAAGCAACGTGTC3' \\
& & R: 5'CGTCGCACAAGCATTTGG3' \\
Smbap-1 & Smp_147920 & MER185277 & F: 5'CGCTACTCACGCTTTACTG3' \\
& & R: 5'CATACTTCCGATGGCAAG3 \\
Smusp-5 & Smp_069960 & MER430357 & F: 5'GCCGAGTTAGAAGTGGATGC3' \\
& & R: 5'GTGTATGGTTGGATTCCTGC3' \\
\hline
\end{tabular}

